\title{
Die Confessio Augustana und die katholische Theologie des 16. Jahrhunderts
}

Die Forderung seit den 1970er Jahren, von katholischer Seite die Confessio Augustana als Ausdruck des Glaubens anzuerkennen, ${ }^{1}$ war eng mit der Entdeckung verknüpft, dass eine solche Anerkennung und Einigung bereits in der katholischen Theologie des 16. Jahrhunderts ihre Vorläufer hatte. Vinzenz Pfnür (19372012) hatte in seiner bei Joseph Ratzinger begonnenen und schließlich bei Erwin Iserloh (1915-1996) eingereichten Dissertationsschrift ${ }^{2}$ über die Rechtfertigungslehre der CA in der katholischen Kontroverstheologie zwischen 1530 und 1535 die Grundlage dafür geschaffen und den Vorschlag einer katholischen Anerkennung derselben begründet, ${ }^{3}$ - die Option für eine traditionskonforme Möglichkeit, die auch (und neben anderen Vertretern) ${ }^{4}$ - zumindest eingeschränkt - von Joseph Ratzinger in der damaligen Debatte aufgegriffen wurde. ${ }^{5}$ Flankiert wurde das ökumenische Postulat hierbei durch eine neue und vertiefte Sicht auf die katholische Theologie des 16. Jahrhunderts, die eben nicht nur in Unverständnis gegenüber der Reformation und in Polemik erstarrt war. Archivalische Entdeckungen zu den Augsburger Vergleichsverhandlungen von Gerhard

1 Vinzenz Pfnür, Die Anerkennung der Confessio Augustana durch die katholische Kirche? Zu einer aktuellen Frage des katholisch-lutherischen Dialogs, in: Internationale katholische Zeitschrift Communio 4 (1975), 298-307; 5 (1976), 374-381, 477 f.; Heiner Grote, Die Augustana-Debatte und die Wiedergewinnung einer Bekenntnisschrift, in: Materialdienst des Konfessionskundlichen Instituts 29 (1978), 26 -34; Peter Gauly, Katholisches Ja zum Augsburger Bekenntnis? Ein Bericht über die neuere Anerkennungsdiskussion, Freiburg i.B. u.a. 1980.

2 Vinzenz Pfnür, Einig in der Rechtfertigungslehre? Die Rechtfertigungslehre der Confessio Augustana (1530) und die Stellungnahme der katholischen Kontroverstheologie zwischen 1530 und 1535, Wiesbaden 1970 (Veröffentlichungen des Instituts für Europäische Geschichte. Abt. Abendländische Religionsgeschichte 60).

3 Pfnür, Anerkennung.

4 Harding Meyer/Heinz Schütte/Hans Joachim Mund (Hg.), Katholische Anerkennung des Augsburgischen Bekenntnisses? Ein Vorstoß zur Einheit zwischen katholischer und lutherischer Kirche, Frankfurt a. M. 1977 (Ökumenische Perspektiven 9).

5 Joseph Ratzinger, Prognosen für die Zukunft des Ökumenismus, in: Ökumenisches Forum. Grazer Hefte für konkrete Ökumene 1 (1977), 31-41; ders., Anmerkungen zur Frage einer „Anerkennung“ der Confessio Augustana durch die katholische Kirche, in: MThZ 29 (1978), 225-237.

๑ OpenAccess. () 2022 Klaus Unterburger, publiziert von De Gruyter. (cc))BY-NC-ND Dieses Werk ist lizenziert unter einer Creative Commons Namensnennung - Nicht kommerziell - Keine Bearbeitung 4.0 International Lizenz. https://doi.org/10.1515/9783110683868-012 
Müller, ${ }^{6}$ Eugène Honée ${ }^{7}$ und Herbert Immenkötter ${ }^{8}$ haben dies bei allen Differenzen untereinander im Grundsatz bestätigt.

Trotz der unbestreitbaren Bedeutung dieser Fragen für den modernen ökumenischen Dialog, ist deren Erforschung dabei doch wieder ins Stocken geraten, ja in vielen Bereichen stagnierte auch die katholische Erforschung der Theologiegeschichte des Reformationszeitalters insgesamt. So kann auch in diesem Referat kein wirklicher Gesamtüberblick geboten werden. Immerhin sollen wichtige Resultate der bisherigen Forschung gesichert und einige neue Akzente gesetzt werden, da der Fortgang in anderen Forschungsfeldern Rückwirkungen auf die Deutung der Stellungnahmen der katholischen Theologie zur Confessio Augustana (= CA) hat. Insgesamt erweist sich die Frage der Stellungnahme zur CA nicht nur als Seismograph für den Fortgang einer theologischen Konfessionalisierung, sondern eröffnet auch wichtige Einblicke für die Deutung der Theologiegeschichte des 16. Jahrhunderts. Terminologisch kämpft dabei auch dieser Beitrag mit der Problematik, dass Begriffe wie „katholisch“, „evangelisch“, „lutherisch“, „altgläubig“ usf. damals in hohem Grade umstritten waren. Unweigerlich läuft man Gefahr, mit ihrer Verwendung einen Grad an konfessionell eindeutiger Abgrenzung in die frühen Jahrzehnte der Reformation zurückzuprojizieren. ${ }^{9}$ Dennoch ist es schwer, die gewohnte Terminologie zu verlassen, da auch die Quellensprache keine eindeutigen Alternativen zu bieten scheint. So bleibt es bei der Problemanzeige, die freilich jeweils mitzubedenken ist.

Im Folgenden soll vor dem Hintergrund der Situation der altgläubigen Kontroverstheologie bis zum Jahr 1530 zunächst die Stellungnahme der katholischen Disputanten in der Confutatio und in den Ausgleichsverhandlungen analysiert

6 Gerhard Müller, Johann Eck und die Confessio Augustana, in: QFIAB 38 (1958), 205-242; ders., Um die Einheit der Kirche. Zu den Verhandlungen über den Laienkelch während des Augsburger Reichstages 1530, in: Erwin Iserloh/Konrad Repgen (Hg.), Reformata Reformanda. FS Hubert Jedin, Bd. 1, Münster 1965, 393-427.

7 Eugène Honée, Die Vergleichsverhandlungen zwischen Katholiken und Protestanten in Augsburg 1530, in: QFIAB 42/43 (1963), 412-434; ders., Die römische Kurie und der 22. Artikel der Confessio Augustana. Kardinal Lorenzo Campeggios Verhalten zur protestantischen Forderung des Laienkelchs während des Augsburger Reichstages 1530, in: Nederlands archief voor kerkgeschiednis. Nieuwe Serie 50 (1969/1970), 140 -196; ders., Die theologische Diskussion über den Laienkelch auf dem Augsburger Reichstag 1530. Versuch einer historischen Rekonstruktion, in: Nederlands archief voor kerkgeschiednis. Nieuwe Serie 53 (1972/1973), 1-96.

8 Herbert Immenkötter, Um die Einheit im Glauben. Die Unionsverhandlungen des Augsburger Reichstages im August und September 1530 [künfig: Einheit], Münster 1973 (KLK 33).

9 Bent Jörgensen, Konfessionelle Selbst- und Fremdbezeichnungen. Zur Terminologie der Religionsparteien im 16. Jahrhundert, Berlin 2014 (Colloquia Augustana 32); Christian Volkmar Witt, Protestanten. Das Werden eines Integrationsbegriffs in der Frühen Neuzeit, Tübingen 2011 (Beiträge zur historischen Theologie 163). 
werden. In einem zweiten Schritt ist zu fragen, welche Rolle die damals erzielten Resultate für Irenik und Polemik in den 1530er und 1540er Jahren gespielt haben, insbesondere für die Religionsgespräche und das Interim. Schließlich ist auf die Frage einzugehen, inwiefern die CA in den Debatten des Trienter Konzils eine Rolle gespielt hat. Am Ende sollen wichtige Entwicklungslinien gesichert werden, die eine theologische Valenz für die Deutung der CA in der Gegenwart haben.

\section{Der Reichstag von 1530: Die katholische Theologie vor der Confessio Augustana}

Für den Augsburger Reichstag lagen eine ganze Reihe von Gutachten bzw. Irrtumslisten vor, die altgläubige Theologen in Auseinandersetzung mit den Schriften der Protestanten erstellt hatten. So hatte König Ferdinand (1503-1564) Gutachten der Theologischen Fakultäten in Tübingen, Freiburg und Wien erbeten $^{10}$ und die bayerischen Herzöge forderten eine solche Stellungnahme von Johannes Eck (1486-1543) ein, der daraufhin seine 404 Artikel zusammenstellte. ${ }^{11}$ An diesen Schriften lassen sich einige Kennzeichen der katholischen (und wohl nicht nur der katholischen) Kontroverstheologie herausarbeiten: a.) sie geht stark additiv vor: Eck etwa sammelt alle Irrtümer aus Exsurge Domine, der Leipziger und der Badener Disputation, usf.; b) sie polemisiert; c) sie reiht die Irrtümer negativ aneinander, ohne den Kontext hinreichend $\mathrm{zu}$ berücksichtigen oder theologisch die Sätze abzustufen, was freilich umgekehrt auch größere Freiheitsräume eröffnete, die gegenteiligen Artikel positiv zu formulieren. Zwei grundsätzliche Tendenzen lassen sich für die katholische Kontroverstheologie vor 1530 ausmachen: Zum einen diejenige, nach den formalen, hierarchischen Autoritäten, päpstliche oder konziliare Entscheidungen und den Konsens der Kirchenväter als Kriterien der Schriftauslegung, zurückzufragen. Zum anderen eine Grundannahme, die die negative Sicht auf die Reformation geprägt hat: Die Rechtfertigungslehre führe bei den einfachen Menschen dazu, die guten Werke zu vernachlässigen. Diese libertinistische Tendenz werde durch lutherische Theologoumena wie dasjenige von der Unfreiheit des Willens noch verstärkt. All dies habe Unordnung, Ver-

10 Remigius Bäumer, Die Lehrunterschiede zwischen Alt- und Neugläubigen im Urteil katholischer Theologen am Vorabend des Augsburger Reichstages, in: Erwin Iserloh (Hg.), Confessio Augustana und Confutatio. Der Augsburger Reichstag 1530 und die Einheit der Kirche, Münster 1980 (RST 118), 189-204, hier 194-196.

11 Wilhelm Gussmann (Hg.), Johann Ecks Vierhundertvier Artikel zum Reichstag von Augsburg 1530. Nach der für Kaiser Karl V. bestimmten Handschrift. Mit zwei Exkursen, Kassel 1930 (Quellen und Forschungen zur Geschichte des Augsburgischen Glaubensbekenntnisses 2). 
achtung der Autoritäten und moralische Verwilderung zur Folge. In diese Sichtweise wirkten vielfach antihussitische Grundeinstellungen ein; die Erfahrungen des Bauernkriegs, der als Folge der Rechtfertigungslehre verstanden wurde, schienen diese Interpretation zu bekräftigen. ${ }^{12}$

Eine solche Auseinandersetzung mit der Reformation lag nun in Augsburg bekanntlich gerade nicht im Interesse des Kaisers und der Ständemehrheit. Dieselben altgläubigen Theologen, die vielfach schon polemisch-literarisch in Erscheinung getreten waren, waren mit dem Einigungswillen des Kaiserhofs und der Ständemehrheit konfrontiert, der schon in der Proposition vom 20. Juni zum Ausdruck kam. ${ }^{13}$ Erste Versuche, die bisherigen Häresiesammlungen auf die Confessio Augustana hin zu aktualisieren, die Responsio theologorum und die Catholica responsio, wurden als zu polemisch verworfen. So mussten die Arbeiten am 16. Juli von vorne beginnen. ${ }^{14}$ Bis zum 3. August wurde nun die Confutatio ausgearbeitet. ${ }^{15}$ Sie unterschied sich von den vorhergehenden Irrtumslisten vor allem dadurch, dass sie streng auf die Confessio Augustana Bezug nehmen sollte, also nicht auch gegen alle anderen, in der Frühphase der Reformation oder sonstwo vorgetragenen Lehren. Sie war ein Gemeinschaftswerk der damaligen führenden katholischen Kontroverstheologen. Die Confutatio sollte kraft der Autorität des Kaisers in seinem Namen verlesen werden und kein Parteidokument sein, ${ }^{16}$ auch wenn Kaiser und Ständemehrheit ihr den Charakter der Endgültigkeit

12 Anselm Schubert, Libertas disputandi. Luther und die Leipziger Disputation als akademisches Streitgespräch, in: ZThK 105 (2008), 411-442, hier 428, 432f., 436; Christoph Volkmar, Reform statt Reformation. Die Kirchenpolitik Herzog Georgs von Sachsen 1488 - 1525, Tübingen 2008 (SMHR 41), 567-569; Remigius Bäumer, Johannes Cochläus (1479-1552): Leben und Werk im Dienst der katholischen Reform, Münster 1980 (KLK 40), 112f.; Gottfried Maron, Bauernkrieg, in: TRE 5 (1993), 319-338, hier v. a. 322f.

13 Karl V., Antragen auf dem Reichstag zu Augsburg 1530, 20. Juni 1530, Hans Eduard Förstemann, Urkundenbuch zu der Geschichte des Reichstages zu Augsburg im Jahre 1530, Halle 1833, Bd. 1, 295-309, hier $308 \mathrm{f}$.

14 Brevis ad singula puncta Confessionis Protestantium Principum Responsio Augustae Catholica et quasi extermporalis Responsio Augustae privatim scripta, Georg Coelestin, Historia Comitiorum anno MDXXX Augustae celebratorum, 4 Bde., Frankfurt (Oder) 1597, hier Bd. 2, fol. $234^{\mathrm{r}}-243^{\mathrm{v}}$; Catholica et quasi extemporalis responsio, Johannes Ficker, Die Konfutation des Augsburgischen Bekenntnisses. Ihre erste Gestalt und ihre Geschichte, Leipzig 1891, 1-140.

15 Herbert Immenkötter, Der Reichstag zu Augsburg und die Confutatio. Historische Einführung und neuhochdeutsche Übersetzung [künftig: Confutatio], Münster 1979 (KLK 39), 25-30.

16 „Das Urteil über Annahme, Modifizierung bzw. Ablehnung der lutherischen Vorlage fällte zunächst auch nach der Confutatio noch der Kaiser. So lebte auch dieser Text de facto noch immer aus dem Anspruch, daß dem Kaiser letztverantwortliche Entscheidung auch in Glaubensfragen zukomme. Erst in den allerletzten Tagen willigten die kaiserlichen Räte ein, die persönlichen Urteile, die dem Kaiser in den Mund gelegt waren, in unpersönliche Formulierungen umzuändern 
nehmen ließen, da die Türkengefahr eine friedliche Verständigung zu verlangen schien. ${ }^{17}$ Trotz deren Bitten verweigerte man den protestierenden Ständen die schriftliche Ausfertigung, um eine langwierige Disputation zu vermeiden, die den Reichstag in den Augen der Altgläubigen zu blockieren drohte. ${ }^{18}$

Dennoch ist die Confutatio ein Dokument der Annäherung, das die Grundlage werden konnte für einen weitgehenden Ausgleich in den Ausschussverhandlungen. ${ }^{19}$ So sah man in den Artikeln 2, 5, 6 und 18 über Erbsünde, Rechtfertigung, gute Werke und freien Willen einen weitgehenden Konsens zur CA. ${ }^{20}$ Ebenso in der Sakramentenlehre der Artikel 8-12. ${ }^{21}$ Bei den Artikeln 1-21 blieben damit noch sieben Differenzpunkte: 1.) Die Kritik an der Definition der Erbsünde, die für ungetaufte Kinder kaum zutreffe und die Begierde zu Unrecht in die Sündendefinition hineinziehe, ${ }^{22}$ 2.) eine sola fide-Lehre, die Liebe und Hoffnung ausklammere, anstatt vom Glauben zu sprechen, der in der Liebe wirksam sei (Gal 5,6), ${ }^{23}$

oder ganz zu streichen. Die Confutatio gab sich in der endgültigen Fassung somit als offizielle altkirchliche Lehre. In Vor- und Nachwort bekannte der Kaiser, daß er sich diese Lehre nach reiflicher Prüfung zu eigen gemacht habe und erinnerte die Zuhörer an die ihm von Gott übertragene Aufgabe, als Vogt und Beschirmer der Christenheit über Annahme und Verbreitung dieser als genuin christlich erkannten Lehre zu wachen.“ Immenkötter, Confutatio, 29.

17 Immenkötter, Einheit, 13.

18 Immenkötter, Einheit, 21-23.

19 Herbert Immenkötter, Die Confutatio - ein Dokument der Einheit, in: Iserloh, Confessio, 205214.

20 Immenkötter, Confutatio, 46f., 49-52, $61 \mathrm{f}$.

21 Vgl. Immenkötter, Confutatio, 53-55.

22 „Aber jene Erklärung des Artikels, die behauptet, die Erbsünde bestehe darin, daß die Menschen ohne Gottesfurcht und ohne Gottvertrauen geboren werden, kann nicht zugelassen werden. Denn es ist doch jedem Christen bekannt, daß ein Mangel an Gottesfurcht und Gottvertrauen eher wirkliche Sünde eines erwachsenen Menschen als Schuld eines Kindes, das eben geboren ist und den Vernunftgebrauch noch nicht hat, genannt werden muss. So sagt der Herr selbst zu Moses: Eure Kinder, die heute noch nicht wissen, was gut und böse ist (Dtn 1,39). Ebenso wird auch die Erklärung der Fürsten verworfen, die behauptet, die Erbsünde sei gleichzusetzen mit der Begierde. Denn sie verstehen die Begierde so, als sei sie eine Sünde, die auch nach der Taufe im Kinde bleibt. [...] Wenn die Fürsten aber mit dem heiligen Augustinus sagen, daß die Erbsünde eine Begierde ist, welche durch die Taufe aufgehoben wird, dann ist dieser Artikel anzunehmen.“ Immenkötter, Confutatio (zu Artikel 2), 47.

23 „Daß hier vom Glauben die Rede ist, kann zugelassen werden - doch so, daß nicht vom „Glauben allein“, wie etliche nicht richtig lehren, gesprochen wird, sondern von dem Glauben, der durch die Liebe wirkt, wie der hl. Paulus an die Galater geschrieben hat (Gal 5,6). “ Immenkötter, Confutatio (zu Artikel 5), 49; „Grundsätzlich kann nicht gebilligt werden, daß die Rechtfertigung so oft ,allein dem Glauben` zugeschrieben wird. Denn die Rechtfertigung ist doch mehr Wirkung göttlicher Gnade und Liebe. So sagt Paulus ausdrücklich: Wenn ich allen Glauben hätte, so daß ich Berge versetzen könnte, und doch die Liebe nicht habe, so bin ich nichts (1 Kor 13,2).“ Immenkötter, Confutatio (zu Artikel 6), 51. 
3.) die Lehre von der Verdienstlichkeit der in der Gnade vollbrachten guten Werke, ${ }^{24}$ 4.) die Wiedergutmachung als integrativer Teil der Buße ${ }^{25}$ und 5.) die Siebenzahl der Sakramente. ${ }^{26}$ Zudem befürchtete man 6.) - trotz des Artikels 8 -, dass die Kirchendefinition des Artikels 7 die Sünder und Unwürdigen nicht als Teil der Kirche sähe. ${ }^{27}$ Als letzter Differenzpunkt blieb 7.) die Frage, ob die Heiligen um ihre Fürsprache angerufen werden durften. ${ }^{28}$ Differenzpunkte waren dann natürlich zudem die abusus-Artikel 22-28. ${ }^{29}$

24 „Wenn aber jemand in diesem Artikel die Verdienste der Menschen, die durch Beistand der göttlichen Gnade geschehen, verwirft, kann dies nicht zugelassen werden. Denn diese Lehre ist manichäisch und nicht christlich.“ Immenkötter, Confutatio (zu Artikel 4), 48; „Hiermit verachtet niemand das Verdienst Christi, sondern wir wissen, daß unsere Werke selbst allein nicht verdienstlich sind, es sei denn aus Kraft des Verdienstes des Leidens Christi. Wir wissen auch, daß Christus der Weg und die Wahrheit und das Leben ist (Joh 14,6). [...] Die gegenteilige Meinung widerspricht also an vielen Stellen dem Evangelium und ist deshalb zu Recht vor elfhundert Jahren, zu den Zeiten des hl. Augustinus, verworfen und verdammt worden.“ Immenkötter, Confutatio (zu Artikel 20), 63.

25 „Der zweite Teil des Artikels aber muß vollends verworfen werden. Dort behaupten sie, es gäbe nur zwei Teile der Buße. Das aber widerspricht doch der ganzen allgemeinen christlichen Kirche, die seit der Zeit der hl. Apostel bis heute gehalten und geglaubt hat, daß es drei Teile der Buße gibt, nämlich die Reue, die Beichte und die Genugtuung.“ Immenkötter, Confutatio (zu Artikel 12), 57.

26 „Der dreizehnte Artikel wird zugelassen und gebilligt, weil sie hier bekennen, daß die Sakramente nicht allein als Erkennungszeichen des christlichen Bekenntnisses, sondern als Zeichen und Zeugnis des göttlichen Willens gegen uns eingesetzt sind. Darum ist aber von ihnen zu verlangen, daß sie all das, was sie hier allgemein von den Sakramenten vorbringen, im besonderen von den sieben Sakramenten der Kirche bekennen und Sorge dafür tragen, daß dies auch ihre Untertanen halten.“ Immenkötter, Confutatio (zu Artikel 13), 59.

27 „Der siebte Artikel des Bekenntnisses behauptet, daß die Kirche eine Versammlung der Heiligen sei. Das kann nicht ohne Gefahr für den heiligen Glauben gebilligt werden. Denn dadurch würden aus der Kirche die Bösen und die Sünder ausgeschieden. Deshalb wurde dieser Artikel auf dem Konstanzer Konzil unter den Irrtümern des Johannes Hus verdammt. Er widerspricht offensichtlich dem heiligen Evangelium. [...] Deswegen kann dieser Artikel auf keinen Fall akzeptiert werde.“ Immenkötter, Confutatio (zu Artikel 7), $52 \mathrm{f}$.

28 „Da nun im einundzwanzigsten und letzten Artikel vorgebracht wird, daß man das Gedächtnis der Heiligen in der Kirche wohl begehen dürfe, auf daß wir ihren guten Werken nachfolgen; daß man aber doch die Heiligen nicht anrufen, noch von ihnen Hilfe begehren soll, da verwundert es, daß die Fürsten und Städte zugelassen und geduldet haben, daß dieser Irrtum, der doch schon so oft in der Kirche verdammt worden ist, nun trotzdem bei ihnen erneut geduldet wird. [...] Denn daß man die Heiligen anrufen darf, ist Überzeugung der ganzen Kirche und wird auch von den heiligen Kirchenvätern Augustinus, Hieronymus, Cyprian, Chrysostomus, Basilius, Bernhard und anderen Doktoren der Kirche bestätigt.“ Immenkötter, Confutatio (zu Artikel 21), $63 \mathrm{f}$.

29 Vgl. Immenkötter, Confutatio, 67-99. 
Eine endlose Debatte mit Schriften und Gegenschriften hierüber sollte nun aber nach dem Willen von Kaiser und Fürsten verhindert werden. Zunächst schlugen einige Fürsten vor, dass der Reichstag einen fürstlichen Vermittlungsausschuss einsetzte. Philipp von Hessen (1504-1567), in dessen Interesse eine weitreichende Vergleichung nicht lag, reiste nunmehr heimlich $\mathrm{ab}^{30}$ und im Ausschuss konnte man sich zunächst nicht verständigen, bis der Kurfürst von Sachsen vorschlug, ein neues Gremium für einen Vergleich in Glaubensfragen einzusetzen. Dies billigten die Ausschussmitglieder, dann auch der Kaiser und die Ständemehrheit. ${ }^{31}$ Am 14. August wurde dieses als Vierzehner-Ausschuss, bestehend aus je drei Theologen [katholisch Johannes Eck, Konrad Wimpina (ca. 1460 1531) und Johannes Cochläus (1479-1552); protestantisch: Philipp Melanchthon (1497-1560), Johannes Brenz (1499-1570) und Erhard Schnepf (1495-1558)], zwei Fürsten und zwei Räten, eingesetzt. ${ }^{32}$ Die kursächsischen Vertreter Melanchthon und Georg Spalatin (1484-1545) entwarfen ein Minimalprogramm für die Stände der CA (Laienkelch, Priesterehe, Abschaffung der Privatmesse und leichte Änderungen am Messformular, Glauben der CA), das die rote Linie darstellen sollte, bis wie weit man nachgeben konnte. ${ }^{33}$ Es stieß freilich im Verlauf der Verhandlungen auf innerprotestantischen Widerspruch, u. a. von Hessen. ${ }^{34}$

In den folgenden Ausschussverhandlungen kam es zu einer weitgehenden Einigung, insbesondere zwischen den Theologen Melanchthon und Eck, aus dessen Feder zwei Gutachten zur CA überliefert sind..$^{35}$ Nicht nur bei der Erbsündendefinition ${ }^{36}$ konnte Einvernehmen hergestellt werden, sondern auch beim

30 Herbert Grundmann, Landgraf Philipp von Hessen auf dem Augsburger Reichstag 1530, Gütersloh 1959 (SVRG 176).

31 Immenkötter, Einheit, 24-28; Gerhard Müller, Die Anhänger der Confessio Augustana und die Ausschußverhandlungen, in: Iserloh, Confessio, 243-257.

32 Eugène Honée, Das Libell des Hieronymus Vehus zum Augsburger Reichstag. Untersuchung und Texte zur katholischen Concordia-Politik, Münster 1988 (RST 125), 55-59.

33 Theologorum scriptum de concordia facienda, [ca.] 15. August 1530, CR 2, $280-285$.

34 Immenkötter, Einheit, 30.

35 Gutachten Johann Ecks über die Confessio Augustana, ca. 4.-10. August 1530, Müller, Johann Eck, 225-239; Bericht Johann Ecks an [Lorenzo Campeggio] über die Verhandlungen des Vierzehner-Ausschusses, ebd. 239-242.

36 „Des anndern artickels ist man vergliechen nach beschehener irer ußlegung unnd declaration, nemlich: Das die erbsundt sey ein mangel ursprunglicher gerechtigkeit, welche dann erforderte glauben, vertrawen unnd gottliche forcht; das auch die begirliche naigung, so uß der erbsundt erwechst, pleyb in dem menschen, aber die erbsundliche schuld welche durch den tauff hingenommen, id est: Quod tollatur quoad formale, sed maneat quoad materiale.“ Honée, Libell, $213 \mathrm{f}$. 
Lohn für in der Gnade vollbrachte gute Werke $^{37}$ und bei der Definition des sola fide, bei der es Eck darum ging, ein libertinistisches, antinomistisches Missverständnis auszuschließen. Eck, der den Glauben ursprünglich als Voraussetzung der Rechtfertigung definieren wollte und vor allem die übernatürliche Liebe als den zentralen Aspekt der Gerechtmachung sah, erklärte sich schließlich damit einverstanden, die sola fide-Lehre zu akzeptieren, wenn vom „lebendigen Glauben“ die Rede sei. Als Einigungsformel akzeptierte man: Quod remissio peccatorum sit per gratiam gratum facientem et fidem formaliter et per verbum et sacramenta instrumentaliter. ${ }^{38}$

Auch das Missverständnis, als lehre die CA nur eine Kirche der Reinen konnte ausgeräumt werden, so dass es einen Konsens in der Ekklesiologie auf dem Boden der CA gab. ${ }^{39}$ Bei den Sakramenten konnte man ebenfalls ein weitreichendes Einvernehmen erzielen. Als Differenzpunkte blieben nur noch bestehen die Frage, ob bei der Buße die Sündenstrafen durch Werke der Genugtuung noch eigens getilgt werden müssen und ob die Heiligen, die im Himmel immer für uns Fürbitte leisten, auch um eine solche angerufen werden dürfen. ${ }^{40}$ Schwieriger gestaltete sich die Einigung in den Artikeln 22-28. Hier hatte sich für Melanchthon und Spalatin Laienkelch, Priesterehe und Abschaffung des Messkanons/der Privatmessen als unverzichtbar herauskristallisiert, während man sonst kompromissbereiter war. ${ }^{41}$ In einem Gutachten für die altgläubigen Fürsten skizzierte Eck

37 „Sovil aber den glauben belangenndt, last man bleiben bey obgemeltem viertten articull unnd desselben declaration. Sovil aber die gute wercke belanget, ist man in deme gleich, das man gute werck wyrcken soll unnd muß unnd das die werck, so uß glauben unnd gnaden gewurcket, Gott gefellig seyendt unnd von der verheißung Gottes wegen belonet werden. Ob aber dieselben werck verdiennstlich, auch ob unnd wie man in dieselben hoffen soll, hatt man sich nit vergleichen konnen: ist zu annderm angestellt." Honée, Libell, 219.

38 Articulus 4, 5, et 6 admittuntur ea declaratione: Quod verbum, sola', ibi, sola fide ' etc., videlicet sola fide iustificiemur, obmittatur et quod remissio peccatorum sit per gratiam gratum facientem et fidem formaliter et per verbum et sacramenta instrumentaliter. Honée, Libell, $214 \mathrm{f}$.

39 Articulus 7. et 8. admittuntur cum declaratione verbi, sanctorum, scilicet: Quod fatentur in ecclesia in hac vita esse malos et peccatores. Honée, Libell, 215.

40 Vgl. Honée, Libell, 215.

41 „Wir haben gestern Ew. Liebden und der anndern anzeigung gehordt unnd unns davon underredt und die sach uffs fleissigst bewogen, konnen doch, verrer disputation hindan gesetzt, kein anndere mittel oder weg bey unns hierinnen bedenncken, dann das man unns die communication sub utraque specie unnd unnsern gaistlichen die ehe, auch bey den messen wie die bey unns gehallten werden, lassen biß zu ferrer hanndlung inn einem gemeinen, frien, cristlichen concilien. Damit aber bey disen artickeln dannocht christlich einigkeit moge erhallten werden unnd die kirch in ordentlichem regiment blyben, dartzu wir zum hochsten geneigt, seind wir erbuttig, uns darnach unnd alßdann, sovil die anndern artickel belangt, von der bischoff gewalt, von unnderscheid der spieß, von gewonlichen kirchen zeremonenn etc., unns jetzunndt hie oder nach- 
zusammen mit Hieronymus Vehus, wie man sich auch in den abusus-Artikeln einigen könne. ${ }^{42}$ So könne man die Priesterehe bis zu einem Konzil, wo sie bereits bestehe, dulden, ${ }^{43}$ ebenso wie die Reichung des Laienkelchs, wenn die Lehre anerkannt würde, dass auch unter einer Gestalt der gesamte Christus gereicht werde und wenn niemand auch zum Empfang des Kelchs gezwungen werde. ${ }^{44}$ Die Kontroverse um den Opfercharakter der hl. Messe sei nur ein „Streit und Zweiung in Worten“. 45

Drei zentrale Fragen blieben zu klären: Laienkelch, Priesterehe und Messopfer (Privatmesse). Dieses waren bereits jene drei Fragen, die Melanchthon Martin Luther (1483-1546) gegenüber als Verhandlungsmasse unmittelbar nach der Verlesung der CA identifiziert hatte. ${ }^{46}$ In Verhandlungen, die er Anfang Juli mit dem Legaten begann, erwartete er, dass in den Fragen von Laienkelch, Priesterehe und Klostergelübden (unter Hintanstellung der Frage der Messe) eine Einigung möglich sei, die es möglich mache, die Jurisdiktion der Bischöfe und des Papstes anzuerkennen. ${ }^{47}$ Der Nuntius hatte die Vorschläge nach Rom weitergeleitet, aber nicht weiter mit ihm verhandelt, sondern die Ausarbeitung der Confutatio unterstützt. ${ }^{48}$ Am 23. Juli erhielt er die erbetene Antwort Roms: Nuntius Tommaso Campeggio (1483-1564) möge den Kaiser drängen, in keinem Punkt nachzuge-

mals, zu gelegen zeit unnd statt, mit den ordinariis locorum zu unndereden und vergleichen, damit inen von unnsern geistlichen gepurend obedientz beesche, und ire geburliche jurißdiction nit verhindert werde unnd sovil muglich unnd cristlich gleichheit in der kirchen in lere unnd ceremonien, gehallten werde.“ Honée, Libell, 222f. - Zur vorherigen Position Melanchthons und Spalatins vgl. Anm. 33.

42 Honée, Libell, 226-233.

43 Honée, Libell, $230 \mathrm{f}$.

44 Honée, Libell, 227-229.

45 „Unnd nachdem im kleinem unnd großen canon mer ein streyt oder zweyung in wortten dann im rechten verstanndt der wortten ,hostia', ,oblatio', sacrificium' oder ,opffer' gewesen, das solliche wort inn cristlichen rechten verstanndt sollen gezogen werden; nemlich: Als Cristus inn dem osterlemblin im alten testament ,figuraliter' oder ,typice‘, das ist bedeutlicher weyß, geopfert und nachmals derselb Cristus am stamen des creutzes sich selbs ein warlich lebendig opffer Gott dem Vatter fur die sunden der menschen geopffert, also wurd jetzundt das, sacrificium‘ oder opffer der messen ein ,misteriale، oder, in misteriis et representativum', das ist ein sacramentlich oder widergedechtlich opffer, teglich inn der kirchen geubet unnd gebraucht, zu erinnerung unnd gedechtnuß der leidenns unnd sterbens Christi.“ Honée, Libell, $229 \mathrm{f}$.

46 Nunc mihi videtur constituendum, priusquam respondeant adversarii, quid verlimus concedere ipsis; de utraque specie, de coniugio, de privata Missa omnis erit deliberatio. De his rebus responde ac inprimis de privata Missa, quam nullo modo videntur amissuri adversarii. Melanchthon an Luther, 26. Juni 1530, WA. Br. 5, 396 f., Nr. 1604.

47 Melanchthon an Campeggio, 6. Juli 1530, CR 2, 168-171, Nr. 761; Scriptum Melanchthons an Campeggio, 5. Juli 1530. CR 2, 246-248, Nr. 819.

48 Honée, Diskussion, $29 \mathrm{f}$. 
ben. ${ }^{49}$ In einer Audienz, die er Melanchthon am 28. Juli gewährte - Melanchthon strebte danach, dass die Verhandlungen mit den katholischen Gegnern auch von Rom gebilligt würden -, erreichte er dann aber die mündliche (und ausweichendlavierende) Zustimmung des Legaten zum Programm einer interimistischen Duldung von Laienkelch und Priesterehe bei Unterwerfung unter die bischöfliche

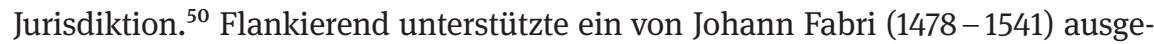
arbeitetes Memorandum den Vorschlag unter Verweis auf die Zugeständnisse des Baseler Konzils gegen die Böhmen.. ${ }^{51}$ Die römische Antwort, die Campeggio schließlich mit dem Kaiser beriet, stellte ein Konzil nur vage in Aussicht und gab zu verstehen, dass man lieber Zugeständnisse des Kaisers in der Kelch- und Zölibatsfrage dulden werde, wenn Karl V. nicht zu einer militärischen Niederwerfung der Protestanten zu gewinnen sei. ${ }^{52}$

Faktisch hatte die römische Positionierung den Weg für eine Einigung mittels disziplinarischer Konzessionen auf Zeit abgesegnet. Bei der Kommunionfrage kam es nun tatsächlich zu einem Teilkonsens; auch bei anderen Fragen wie der Priesterehe zeichnete sich der Weg einer interimistischen Duldung des status quo an. ${ }^{53}$ Zur Annäherung in den Artikeln 22-28 meldeten freilich Hessen, Lüneburg und Nürnberg Widerspruch an. ${ }^{54}$ Da noch immer eine Hoffnung auf Einigung bestand, billigte der Kaiser die Einsetzung eines neuen Sechser-Ausschusses. In ihm waren je zwei Juristen und ein Theologe, erneut standen sich so Melanchthon und Eck gegenüber. ${ }^{55}$ Beide Seiten forderten sich auf, neue Vermittlungsvorschläge zu machen und immer wieder erklärte Eck, es gebe letztlich keine sachlichen, sondern nur noch terminologische Differenzen, besonders bei dem noch verbliebenen kleinen Rest an umstrittenen Glaubensgegenständen: Wiedergutmachung bei der Beichte; Anrufung der Heiligen; Verdienstlichkeit der guten Werke der Gerechtfertigten im Gnadenstand. ${ }^{56}$ Im Sechser-Ausschuss arbeitete man vor allem mit der Denkfigur der Duldung bis zum nächsten Konzil, etwa, dass die Altgläubigen in protestantischen Gebieten den Laienkelch und die Priester-

49 Honée, Kurie, 160 - 171.

50 Honée, Kurie, 171-180.

51 Honée, Kurie, 180 - 184; Müller, Einheit.

52 Honée, Kurie, 184-190. Disziplinarische Zugeständnisse seien möglich, nur wolle man von den Protestanten die Unterschrift unter die Unionsbulle Laetentur coeli des Konzils von Florenz.

53 Honée, Libell, 222-253.

54 Immenkötter, Einheit, 51-56.

55 Valentin von Tetleben, Protokoll des Augsburger Reichstages von 1530, hg.v. Herbert Grundmann, Göttingen 1958 (Schriftenreihe der Historischen Kommission bei der Bayerischen Akademie der Wissenschaften 4), $130 \mathrm{f}$.

56 Vortrag der päpstlichen Partei im Ausschuss der Sechs, 24. August 1530, in: Förstemann, Urkundenbuch, Bd. 2, 292-298, Nr. 163. 
ehen duldeten, oder die Protestanten die Klöster oder den Messkanon, soweit die applicatio missae gestrichen würde. ${ }^{57}$ Hieronymus Vehus (1484-1544), Kanzler des Markgrafen von Baden, hatte von Seiten der Altgläubigen angeboten, dass die gesamte Messfrage bis zu einem Konzil unentschieden bleiben solle; ${ }^{58}$ gerade der interimistische Verzicht auf die Applikationslehre wurde von altgläubiger Seite als erhebliches Zugeständnis aufgefasst, um einen Frieden im Reich und so die Abwehr gegen die Türken zu sichern. Obwohl Eck selbst von der Messopferlehre überzeugt war, konnte er dieses Zugeständnis unterstützen, da auch für ihn letztlich die gesamte mittelalterliche Stiftungspraxis nicht zum strikten Glaubensgut gehörig galt (sondern Teil des Streits um die rechte zeremonielle Praxis war) und deshalb diskutabel war. ${ }^{59}$ Melanchthon hatte auf der anderen Seite sein „Bedenken“ mit fünf möglichen Zugeständnissen abgefasst; ${ }^{60}$ auf dieser Grundlage wäre die Einigung möglich gewesen. Doch nun zerbrach endgültig die Einheit im protestantischen Lager, da vielen eine solche interimistische Unterwerfung unter die bischöfliche Jurisdiktion unannehmbar schien. ${ }^{61}$ Die sechs Verordneten einigten sich noch auf die Abfassung eines zusammenfassenden Berichts, doch trotz vielfachen Drängens war die protestantische Seite nun zu keinen weiteren

\section{Honée, Libell, $256-263$.}

58 „Die Messen belangend sollten sy in offnen (gemeinen) und privatmessen den merern und minderen canonem halten, dwyl doch nichts böses darinnen. Deß sy zwyfflelig hielten, so fast uff dryen puncten stünde, die nunmehr zu gutten verstand gelüthert werden, als des priesters uffopferung in person gemeiner kirchen, die ettliche gedechtnüß der heylgen und comunion oder niessung des sacraments vom priester und anderer. Aber die anderen disputierliche stuck, als de merito, de opere operato et applicatione misse - als von verdienstlichen wercken der messen, vom werck so des orts gewürckt, und wie die messen einem anderen zugeeygnet werden - und derglichen disputierlichen stuck, hett man zu entscheydung des concilii anzustellen. Item das auch die privatmessen nit abgethan wurden." Honée, Libell, 258.

59 Deinde per amorem Christi a te peto, ut modis quibuscunque potes, in utilitatem Germaniae et omnium Principum concordiae rationes quaerere et inire studeas, quo ecclesia vicissim in unum coprus coalescat. Rationes disputabiles remittantur ad concilium. Quid de applicatione missae et opere operato dimiens? Ego quidem eius rei tam certus sum, ut vel morte mea id testari non dubitem, et tamen pacis retinendae causa omnibus ordinibus suadeo, ut indecisa ad concilii iudicium reiiiciatur. Coram omnibus principibus et dominis vestris his de rebus loqui mallem. Igitur cum collegis tuis exemplum imitare. Sic fiet bona pax et tranquillitas, ac veniet gladius super Turcas. Eck an Melanchthon, 27. August 1530, CR 2, $316 \mathrm{f}$.

60 Gutachten Melanchthons, 24. August 1530, in: Karl Schornbaum, Geschichte des Reichstages von Augsburg im Jahre 1530, in: ZKG 26 (1905), 142-149, hier 144-146.

61 „Auf solichs alles ist bei Lunenburg, Hessen und uns von Städten nicht für gut angesehen, sich darauf mit dem Widertheil weiter einzulassen. [...] Und haben sich gleichwohl zwischen dem Lunenburgschen Canzler und dem Philippo etliche widerwärtige Reden begeben. [Ist] ihme auch, dem Philippo, von den Hessischen, nämlich dem Schnepfen, ihren Predigern Widerpart gehalten worden.“ Gesandte Nürnbergs an den dortigen Rat, 26. August 1530, CR 2, 312-314, hier 313. 
Verhandlungen bereit. ${ }^{62}$ Auch letzte Sonderverhandlungen des badischen Kanzlers Vehus, die dieser auf Drängen Ferdinands führte, und in denen er eine Duldung des protestantischen Status quo bis zu einem künftigen Konzil zugestehen wollte, fanden keine allgemeine Akzeptanz mehr bei den Ständen der CA. ${ }^{63}$

$\mathrm{Zu}$ konstatieren bleibt: mit Eck, Cochläus, Fabri und Wimpina haben führende altgläubige Kontroverstheologen eine Einigung in den Glaubensartikeln der CA für möglich gehalten. In der Rechtfertigungs- und Sakramentenlehre waren es wenige Teilfragen, die noch strittig waren, bei denen man aber hinter terminologischen Differenzen gemeinsame Anliegen ausmachen konnte. Weder vertrat die katholische Seite - wie unterstellt - die pelagianisierenden Glaubenssätze, die man aus Gabriel Biel (ca. 1410 -1495) hätte schöpfen können, noch die protestantische Seite eine libertinistisch-antinomistische Rechtfertigungslehre. In Fragen der Ekklesiologie und des kirchlichen Amts sah man keine entscheidenden Differenzen, doch wohl auch ein Indiz dafür, dass CA 5 und CA 14 keinen Widerspruch zu den katholischen Ämtern, die freilich nicht Glaubensgegenstand waren, sondern im Kirchenrecht ihre Begründung fanden, anmelden wollten. Die Differenz blieb vor allem eine zeremonielle: beide Seiten sahen auf der Gegenseite abusus. Hier konnten Teilkonsense erzielt werden und eine wechselseitige Duldung des Unverglichenen stand im Raum. Der Glaubensstreit, so kann man konstatieren, war auf der Basis der CA nach den führenden katholischen Kontroverstheologen somit noch nicht unbedingt ein Gegensatz zwischen zwei unvereinbaren Grundprinzipien, sondern das Ringen zwischen zwei zeremoniellen Ausgestaltungen des einen Glaubens, die teilweise im Widerspruch zueinanderstanden.

\section{Die Confessio Augustana im Fortgang der Kontroverse}

Der in Augsburg erzielte weitgehende Konsens stand freilich unter der hermeneutischen Voraussetzung, dass die CA wirklich authentisch den Glauben der Protestierenden wiedergebe. Die altgläubigen Theologen waren ja ausdrücklich angewiesen worden, nur auf diese zu reagieren und nicht Invektiven gegen alle

62 Honée, Libell, 254-280; Immenkötter, Einheit, 64-66.

63 Honée, Libell, 281-346; ders., Kontinuität und Konsistenz der katholischen Concordiapolitik während des Augsburger Reichstages von 1530, in: Herbert Immenkötter/Gunther Wenz (Hg.), Im Schatten der Confessio Augustana. Die Religionsverhandlungen des Augsburger Reichstages 1530 im historischen Kontext, Münster 1997 (RST 136), 84-97; Immenkötter, Einheit, 68-80. 
möglichen, in den zehn Jahren davor geäußerten Meinungen einfließen zu lassen. Deshalb schieden sich an diesem Punkt die Geister. Hielt man an der Grundvoraussetzung fest, dann war eine Einigung nach wie vor möglich. Der Kern der Spaltung lag - so die eine Position - in Missbräuchen, so dass eine entschiedene katholische Reform den Protestantismus regelrecht überflüssig machen konnte, jedenfalls aber den Boden für eine künftige Einigung zu bereiten schien. Die Theologen, die dieses mit der CA letztlich korrespondierende Konzept vertraten, waren die sog. Vermittlungstheologen. Daneben gab es andere Theologen, die sich von dieser Hermeneutik lösten. Sie kritisierten weniger die Confessio Augustana in sich; nur glaubten sie nicht, dass diese vollständig und korrekt die protestantischen Prinzipien wiedergebe. Sie sei eher Ausdruck eines Täuschungsmanövers. In den Jahren 1520 - 1522 haben Luther und Melanchthon ihr wahres Gesicht gezeigt; eine seither angeblich erfolgte Mäßigung sei nichts als der heimtückische Versuch, den Gegnern Sand in die Augen zu streuen. Dies war explizit die Position von Johannes Cochläus in seinen Philippicae. ${ }^{64}$

Innerhalb dieses Gegensatzes, das hat Vinzenz Pfnür am Beispiel der Rechtfertigungslehre gezeigt, ist die katholische Position in Bezug auf die CA in den 1530 Jahren nicht uniform. Sola fide im Sinn eines nackten Glaubens, der Buße, Hoffnung, Liebe und gute Werke ausschließe, wurde abgelehnt. Nicht die CA stand inhaltlich im Zentrum der Kritik, sondern die Reformatoren und die angeblich demoralisierenden Konsequenzen ihrer Rechtfertigungslehre im Volk. ${ }^{65}$ Man kann also sagen, dass das Scheitern der Verhandlungen auf dem Augsburger Reichstag dazu geführt hat, dass die wechselseitigen Befürchtungen, die dort weitgehend ausgeräumt waren, wieder eine stärkere Bedeutung erlangten. Dennoch blieb ein Konsens möglich, in Fragen der Sünde und Rechtfertigung etwa im Leipziger Religionsgespräch von 1534, wo v. a. Melanchthon und der Dominikaner Michael Vehe (1485-1539) verhandelt hatten. ${ }^{66}$

64 Johannes Cochläus, Philippicae quatuor in apologiam Philippi Melanchthonis ad Carolum V. Imperatorem Romanorum, pium, foelicem, triumphatorem, semper Augustum, Leipzig 1534.

65 Pfnür, Einig, 115-118.

66 „Also haben wir die Articul vorgenommen vom ersten, vom andern, das ist von der Erbsünde, vom dritten, sind wir einträchtig. Darnach sind wir kommen auf die Articul vom Glauben und vom Verdienst; davon haben wir lang disputirt, und hab ich declarirt, was unsre Lehre sey, und wie es zu verstehen, daß wir sagen sola fide, daß die Person allein um Christus Willen gewißlich Gott gefalle; und darnach muß der Gläubige vor Gott auch solche Werke der Gerechtigkeit haben, daß er ein gut Gewissen habe, obgleich die Person Gott um anderer Ursach willen gefället. Und wiewohl D. Vehe solches nicht anficht, so wollt er doch meine Stellung der Vergleichung nicht annehmen, denn er steckt in seiner Phantasey, daß er Gnad und Gerechtigkeit deutet nicht: Gottes gnädigen Willen und Gunst, sondern: unsre Verneuerung in uns. Er stellte etliche Form, die ich auch verwarf, und noch die Copien bei mir habe. Endlich stellet er diese Form in Latein, die ich 
Die Überzeugung, dass die Protestanten in der CA eine gemeinsame Glaubensbasis nur vorgeheuchelt hätten, war jedenfalls bei den katholischen Theologen nicht allgemein. Anfang 1540 hatte der Wiener Hoftheologe Friedrich Nausea (1496-1552) ein Gutachten für König Ferdinand verfasst, ob die CA eine geeignete Grundlage für anstehende Religionsgespräche wäre. Er rät, sich auf die CA $\mathrm{zu}$ stützen und ihr $\mathrm{zu}$ folgen und rezipierte dabei die Augsburger Ausgleichsverhandlungen. ${ }^{67}$ Im Detail sah er mehr Divergenzen, als letztlich in Augsburg übrig geblieben waren und folgte so in etwa der Position der Confutatio. Entsprechend der Hermeneutik der CA und der Augsburger Verhandlungen sah auch er die Hauptunterschiede in den Missbrauchsartikeln, wo er zu einer gewissen Nachgiebigkeit den Protestanten gegenüber riet, vor allem was den Laienkelch und die Priesterehe anging, nicht aber in der Messopferlehre. ${ }^{68}$ Dass auch von den katholischen Kontroverstheologen, die eine klare Abgrenzung von den Reformatoren wollten, nicht der Inhalt der Confessio Augustana kritisiert wurde, zeigt das Beispiel Johannes Fabris, der von König Ferdinand im Zusammenhang

jetzt verdeutscht habe, wie folget: Die Gerechtigkeit wird erlangt ohne vorgehende Verdienst der Reue oder Glaubens, oder Lieb oder Hoffnung, oder ander Werk, sondern widerfährt uns durch Gottes Gnad allein, die in Gotteswerk, so er uns gerecht macht, wirkt erstlich in uns Glauben, dadurch wir an Gott, der uns gerecht macht, glauben, und fest an seinem Wort und Verheißung der Gnade hangen. Durch diesen Glauben aber wirket Gott in uns die Liebe, dadurch wir Gerechtigkeit lieben und Unrechtes hassen. Auch wirkt er Hoffnung, dadurch wir vertrauen auf seine Barmherzigkeit, durch welche allein die erschrocken Gewissen getröstet werden. Und lehren diejenigen recht, so in diesem Verstand sprechen, daß der Glaube allein gerecht mache, nämlich daß sie von der Gerechtmachung ausschließen Verdienst der Lieb und ander Werk, und sagen, daß allein Gnade und Barmherzigkeit uns gerecht mache. Aber gute Werke von Gott gebothen muss man tun, nicht daß Gerechtigkeit nicht umsonst um Christi willen erlangt werde, sondern darum, daß Gott gute Werke gebothen hat, daß auch Gerechtigkeit und Glaube nicht bleiben kann ohne gute Werke, und ist noth, die sündliche Lust in unserm Fleisch mit guten Werken zu tödten, daß die Verneuerung des Geistes zunehme. ' Dieses, wie erzählt, ist der Articul, wie er vom andern Theil gestellet; und wiewohl wir dennoch Bedenken hierin gehabt, die wir auch angezeigt; haben's aber nicht [i.e. so haben wir doch nicht] weiter gestritten, sondern also für uns diesmal diese Form für leidlich bleiben lassen.“ Melanchthon an Kurfürst Johann Friedrich, 3. Mai 1534, CR 2, 722-726, Nr. 1187, hier 722f. Danach scheiterten die Gespräche an der Frage des Messopfers. 67 Herbert Immenkötter, Friedrich Nausea und die Augsburger Religionsverhandlungen, in: Remigius Bäumer (Hg.), Reformatio ecclesiae. Beiträge zu kirchlichen Reformbemühungen von der Alten Kirche bis zur Neuzeit. FS Erwin Iserloh, Paderborn u.a. 1980, 467-486, hier v.a. 473 und $483 \mathrm{f}$.

68 Immenkötter, Nausea, 477-482. 
der Religionsgespräche 1540/41 um Gutachten gebeten wurde. Das Problem sei gerade, das Luther und Melanchthon etwas anderes lehrten als die CA. ${ }^{69}$

Allmählich schieden sich so innerkatholisch die Fronten: Auf der einen Seite standen die Vermittlungstheologen, Julius Pflug (1499-1564), Johannes Gropper (1503-1559), Georg Witzel (1501-1573) und Georg Cassander (1513-1566). Für Sie war die Konzeption maßgebend, dass zwischen Katholiken und Protestanten keine unüberbrückbaren Lehrdifferenzen standen, sondern v.a. Fragen der zeremoniellen Praxis. ${ }^{70}$ Sie traten deshalb für die Gewährung von Priesterehe und Laienkelch ein. Auf der anderen Seite standen etwa die frühen Jesuiten, Petrus Faber (1506 - 1546), Jerónimo Nadal (1507-1580), Petrus Canisius (1521-1595): für sie war die CA Ausdruck des „wankelmütigen“ Melanchthons, die nicht das wahre Denken der Reformatoren widerspiegle. ${ }^{71}$ Primär wurde deshalb nicht deren Inhalt kritisiert, für eine Einigung mit den Protestanten musste sie freilich ungeeignet bleiben, da Melanchthon dissimuliert habe. Bei Johannes Eck finden sich beide Dimensionen: er unterschied eine Disputation, die den Gegner besiegen und widerlegen möchte, was ein unparteiischer Schiedsspruch dann aussprechen sollte, von einem amicum colloquium, das hinter terminologischen Differenzen das gemeinsame Anliegen herausarbeiten möchte. ${ }^{72}$ Zwischen den beiden Rollen

69 Vinzenz Pfnür, Die Einigung bei den Religionsgesprächen von Worms und Regensburg 1540/41 eine Täuschung?, in: Gerhard Müller (Hg.), Die Religionsgespräche in der Reformationszeit, Gütersloh 1980 (SVRG 191), 55-88, hier 56-66.

70 John Patrick Dolan, The Influence of Erasmus, Witzel and Cassander in the church ordinances and reform proposals of the United Duchees of Cleve during the middle decades of the 16th century, Münster 1957 (RST 83); August Franzen, Zölibat und Priesterehe in der Auseinandersetzung der Reformationszeit und der katholischen Reform des 16. Jahrhunderts, Münster 1969 (KLK 29), 42-63; ders., Die Kelchbewegung am Niederrhein im 16. Jahrhundert. Ein Beitrag zum Problem der Konfessionsbildung im Reformationszeitalter, Münster 1955 (KLK 13); Barbara Henze, Aus Liebe zur Kirche. Die Bemühungen Georg Witzels (1501-1573) um die Kircheneinheit, Münster 1995 (RST 133); Johannes Meier, Johannes Gropper (1503-1559) - Theologie für eine Erneuerung der Praxis der Kirche, in: AmrhKG 70 (2018), 127-146; Peter Walter, Julius Pflugs gelehrtes Umfeld, in: Dialog der Konfessionen. Bischof Julius Pflug und die Reformation, Petersberg 2017 (Schriftenreihe der Vereinigten Domstifter zu Merseburg und Naumburg und des Kollegiatstifts Zeitz 10), 69-87.

71 Jos E. Vercruysse, ,Melanchthon, qui modestior videri voluit .... Die ersten Jesuiten und Melanchthon, in: Günter Frank (Hg.), Der Theologe Melanchthon, Stuttgart 2000 (MelanchthonSchriften der Stadt Bretten 5), 393-409; Alfons Knoll, Philipp Melanchthon aus römisch-katholischer Sicht, in: Michael Fricke/Matthias Heesch (Hg.), Der Humanist als Reformator. Über Leben, Werk und Wirkung Philipp Melanchthons, Leipzig 2011, 311-344, hier 313-326.

72 Vinzenz Pfnür, Excommunicatio und amicum colloquium. Das Religionsgespräch auf dem Reichstag zu Augsburg 1530 auf dem Hintergrund der Frage des Lutherbannes, in: Wolfgang Beinert/Konrad Feiereis/ Hermann Josef Röhrig (Hg.), Unterwegs zum einen Glauben. FS Lothar Ullrich, Leipzig 1997 (Erfurter theologische Studien 74), 448-460. 
konnte er bereits 1530 wechseln. Er stand den Religionsgesprächen in Worms und Regensburg und auch dem im Regensburger Buch Erreichten skeptisch gegenüber, ließ sich aber doch auch dann noch auf den Versuch eines Vergleichs mit Melanchthon auf der Basis der CA ein und hielt diese nach wie vor für eine Basis, auf der eine Einigungsformel im Glauben möglich sei. ${ }^{73}$ Trotz der ablehnenden Haltung der bayerischen Herzöge stand Eck so hinter der Einigung in der Erbsündenlehre in Worms, wobei die CA hier eine wichtige Basis bildete. Während Ecks Gutachten von Ende 1540 eine Einigung auf der Grundlage der Recht verstandenen Confessio Augustana für möglich hielt (am schwierigsten schien ihm die Frage des Messopfers bzw. der Privatmesse zu lösen), tilgte das Regensburger Buch seiner Meinung nach zahlreiche Formulierungen, für die bereits eine Einigung erzielt worden sei. ${ }^{74}$

Dennoch wirkten die Konzeption der Confessio Augustana und die Augsburger Vergleichsverhandlungen weiter. Dies lässt sich noch in der Erarbeitung der Interimsformel - den wichtigsten Anteil hatten auf altgläubiger Seite Julius Pflug und Michael Helding (1506-1561) - erkennen, wo genau in den drei hauptsächlich in Augsburg strittigen Gebieten den Protestanten Zugeständnisse gemacht werden sollten. ${ }^{75}$ Laienkelch - Priesterehe ${ }^{76}$ - Verzicht auf Privatmessen bzw. eine Messopfertheologie, die darauf verzichtete, von einer sündenvergebenden Funktion der Messe zu sprechen bzw. von einer Unabhängigkeit und Eigenständigkeit des Opfers der Kirche gegenüber dem Kreuzesopfer. ${ }^{77}$ Das Interim war sozusagen ein Substrat, ein Minimum an Zugeständnissen, das man mit katholischer Brille aus der beinahen Einigung in Augsburg 1530 herausziehen konnte; so konnte etwa Georg Witzel in seiner „Bestendigen Antwort“ mit Unverständnis über den Widerstand auf protestantischer Seite reagieren, ähnlich wie später Julius Pflug. ${ }^{78}$

Blieb so bei den Religionsgesprächen im Reich die CA präsent, sei es, dass sie von altgläubiger Seite als mögliche Basis einer Verständigung genommen wurde, sei es, dass sie als Täuschungsmanöver galt, das die Häresien der Reformatoren

73 Pfnür, Einigung, 71.

74 Pfnür, Einigung, 71f.

75 Peter M. Seidel, Michael Helding (1506-1561). Ein Bischof im Dienst von Kirche und Reich, Münster 2012 (RST 157), 63-75.

76 Das Augsburger Interim von 1548. Deutsch und lateinisch, hg.v. Joachim Mehlhausen, Neukirchen-Vluyn 1970, Art. 26, hier $142 \mathrm{f}$.

77 Mehlhausen, Interim, Art. 22, 102-123 und Art. 24, $134 \mathrm{f}$.

78 Georg Witzel, Bestendige Antwort wider der Luterischen Theologen Bedencken, welchs sie widers Interim Geschrieben, Köln 1549; Julius Pflug, Christliche Ermanu[n]g an des Naumburgischen Stieffts vnderthanen vnd vorwandten, wes sie sich bey dem vorgefallenem hochbeschwerlichem mißvorsta[n]d in Religions sachen halten sollen / Damit sie den dinge[n] jhnen selbst zu hochstem nachtheil nicht zu viel oder zu wenig thuen, Köln 1562. 
nur verschleiere, so gilt das für das Trienter Konzil nur abgeschwächt und indirekt. Zu schwach war die Beteiligung aus dem Reich, so dass Theologen, die noch direkt in die Verhandlungen um diese involviert waren, kaum eine Rolle spielten. Johannes Fabri hatte zwar schon 1536 in einer Denkschrift angemahnt, ein künftiges Konzil müsse sich mit den Schriften der Reformatoren befassen, so dass diese im Vorfeld erworben und eingehend studiert werden müssten. ${ }^{79}$ Das päpstliche Rom war an den Originalschriften jedoch wenig interessiert und hielt Bücherlisten für ausreichend. ${ }^{80}$ Als das Konzil dann 1546 mit der Ausarbeitung von Lehrdekreten begann, wurde eine direkte und negative Verurteilung der Protestanten zunächst vermieden, was von einigen Konzilsvätern kritisiert wurde. Bei der Rechtfertigungsdebatte erklärten die Kardinäle Pole und Pacheco entschieden, die Reformatoren müssten hierzu im Original studiert werden, ${ }^{81}$ doch nur eine Minderheit schloss sich ihnen an. Erst ab der Sakramentendebatte führte der Auftrag des Konzilspräsidenten Marcello Cervini (1501-1555) dazu, dass in den vorbereitenden Theologenkommissionen Irrtumslisten aus dem protestantischen Schrifttum erstellt wurden. Zu den Referenzwerken, die vor allem von den Jesuitentheologen nun beigezogen wurden, zählten auch die CA und die Apologie. ${ }^{82}$ So rekurrierte man dabei auf Formulierungen der CA, die gegen das Fronleichnamsfest gerichtet waren oder die die Sakramentengnade vom Glauben scheinbar abhängig machten. ${ }^{83}$

79 Johannes Fabri, Praeparatoria futuri nuper indicti universalis cconcilii, 6. Juli 1536, CT IV, $10-26$.

80 Theobald Freudenberger, Zur Benützung reformatorischen Schrifttums in Trient, in: Remigius Bäumer (Hg.), Von Konstanz nach Trient. Beiträge zur Geschichte der Kirche von den Reformkonzilien bis zum Tridentinum, München/Paderborn/Wien 1972, 577-601, hier 580-584.

81 Pedro Pacheco de Villena, Debatte 21. Juni 1546, CT I, 82, 19-32; Illud autem velim a patribus observari, ut libros omnes, etiam adversariorum, non ut adversariorum, sed ut cuiuslibet alterius, legant, et nolint protinus dicere: id Lutherus dixit, ergo falsum est; nam mos est et semper fuit haereticorum, ut vera falsis immisceant, ut eorum mendacia magis credibiliora suis sequacibus faciant; quod ni facerent, eorum libros nullus legeret. Reginald Pole, Debatte 21. Juni 1546, CT I, 82, $38-43$.

82 Congregatio generalis. Referuntur errores circa sacramenta in genere, baptismum et confirmationem, ac impedimenta residentiae, 17. Januar 1547, CT V, 835-838.

83 Confessione Augustana articulo 5.: Per verbum et sacramenta tamquam per instrumenta donatur Spiritus Sanctus, qui fidem efficit, ubi et quando visum est Deo, in iis, qui audiunt evangelium. Congregatio generalis 836, $20-22$ (= CA 13,2); Nullum festum magis odi quam festum corporis Christi. Idem in confessione Augustana. Articuli haereticorum super sacramento Eucharistiae propositi examinandi theologis minoribus, 3. Februar 1547, CT V 869-871, hier 870, 6 (= CA 22,12); In confessione Augustana cap. de utraque specie: Hic mos communicandi sub utraque specie habet mandatum Domini. Matth. 26 [27]: „Bibite ex hoc omnes“, ubi manifeste praecipit Christus, ut de poculo omnes bibant. Congregatio generalis $870,15-18$ (= CA 22,1). 
Freilich war auch die mit der CA verbundene Hermeneutik der Einheit in Glauben und der Differenz in den abusibus dem Konzil nicht völlig fremd; schon zu Beginn forderte ja die kaiserliche Seite, die Reform der Kirche zu behandeln und die Glaubensfragen bis zum Eintreffen der Protestanten zurückzustellen, damit man hierüber dann gemeinsam eine Antwort finden könne. ${ }^{84}$ Als kaiserlicher Orator war am 30. August 1551 Friedrich Nausea auf dem Konzil eingetroffen, wo er die Gewährung des Laienkelchs forderte. ${ }^{85}$ In der dritten Sitzungsperiode waren es dann König Ferdinand I., der bayerische Herzog Albrecht V. (1528-1579) und Herzog Wilhelm von Cleve (1516-1592), die Reformprogramme postulierten, zu denen die Gewährung von Laienkelch, Priesterehe und Fastendispensen gehörten. ${ }^{86}$ Solche Forderungen sind natürlich nicht einfach eine Reminiszenz an die CA. Sie verbanden sich jedoch mit Strömungen des Erasmianismus und des Evangelismus in Italien und wurden auch von Papst Pius IV. (1499/1558-1565) unterstützt, so dass es immerhin zu päpstlichen Kelchindulten kam. Dadurch wurde der Papst und führende Exponenten des Konzils wie Giovanni Morone (1509-1580) so aber einer extrem rigorosen, antiprotestantischen Partei suspekt, so dass der Kardinalinquisitor Michele Ghislieri (1504-1572) Häresieprozesse gegen beide projektierte. ${ }^{87}$

84 Hubert Jedin, Geschichte des Konzils von Trient. Bd.1-4/2, Freiburg i.B. 1949-1975, hier Bd.1, 179-182; 202f., Bd. 2, 22-26.

85 Friedrich Nausea, Ad interrogata super sequentibus de sacrosancto eucharistiae sacramento articulis in oecumenico concilio Tridentino responsio, 21. September 1551, CT VII,2, 154-171, hier $160-169$.

86 Alfons Beckenbauer, In geistlichen Sachen unterwegs. Die Missionen des Landshuter Kanzlers Augustin Baumgartner im Auftrag Herzog Albrechts V., in: VHVN 108 (1982), 15-39; Karl Saftien, Die Verhandlungen Kaiser Ferdinand I. mit Papst Pius IV. über den Laienkelch und die Einführung desselben in Österreich. Mit archivalischen Beilagen, Göttingen 1890; Ernst Laubach, Ferdinand I. als Kaiser. Politik und Herrscherauffassung des Nachfolgers Karls V., Münster 2001, v. a. 359-516; Jedin, Geschichte, Bd. 4/2, 155-173.

87 Elena Bonora, Morone e Pio IV, in: Massimo Firpo/Ottavia Niccoli (Hg.), Il cardinale Giovanni Morone e l'ultima fase del concilio di Trento, Annali dell'Istituto storico italo-germanico in Trento, Bologna 2010 (Quaderni 80), 30 - 42; Massimo Firpo, Inquisizione romana e Controriforma. Studi sul cardinal Giovanni Morone (1509-1580) e il suo processo d'eresia, Brescia 22005; Elena Bonora, Giudicare i vescovi. La definizione dei poteri nella Chiesa postridentina, Rom/Bari 2007 (Quadrante Laterza 137); dies., La controriforma, Rom/Bari ${ }^{4} 2008$ (Biblioteca essenziale Laterza 35); Claus Arnold, Die römische Zensur der Werke Cajetans und Contarinis (1558-1601). Grenzen der theologischen Konfessionalisierung, Paderborn 2008 (Römische Inquistion und Indexkongregation 10). 


\section{Fazit}

Der altgläubige Rekurs auf die Confessio Augustana wurde im Lauf der 16. Jahrhunderts schwächer. Die Option der Einigung auf ihrer Grundlage rückte ins Irreale. Dennoch gilt es festzuhalten, dass die CA für die führenden katholischen Kontroverstheologen durchaus als brauchbare Grundlage einer Verständigung im Glauben erschien; lehnte man dies ab, dann weniger wegen des Inhalts des Bekenntnisses an sich, sondern da man glaubte, dass es die wahren Intentionen Melanchthons und Luthers nur verharmlosend zum Ausdruck bringe.

Dort weil die CA als möglicher Referenzpunkt des gemeinsamen Glaubens akzeptabel erschien, lassen sich aber drei für die Theologiegeschichte des 16. Jahrhunderts bedeutsame Entwicklungslinien herausarbeiten:

1. Die Suche nach einem gemeinsamen Anliegen hinter differenten, scheinbar gegensätzlichen Formulierungen ist nicht ein neuer, interessegeleiteter Versuch der Uminterpretation der modernen Theologie, sondern bereits der Standpunkt der Akteure des 16. Jahrhunderts, so explizit ja Johannes Ecks.

2. Eine Sichtweise auf die Reformationsgeschichte, nach der von Beginn an gravierende theologische Unterschiede jede Verständigung unmöglich gemacht hätten, ist sicher unzutreffend. Vielmehr glaubten nicht nur zahlreiche katholische Landesherrn, dass durch Reformen Missstände abgestellt und dem Hass auf den Klerus die Grundlage entzogen werden müsse. ${ }^{88}$ Dies war letztlich auch das Konzept der Confessio Augustana. Es ging also vor allem um den Streit um Zeremonien, während - beim anderen guter Wille vorausgesetzt - in den Fragen von Theologie und Rechtfertigung eine Verständigung möglich erschien.

3. Besonders unproblematisch erschien eine mögliche Einigung in den Fragen des kirchlichen Amts und der Ekklesiologie. Während im 20. Jahrhundert die Theologen und Historiker beider Konfessionen hier häufig den tiefsten Unterschied zwischen den Konfessionen ausmachten und ein enges Implikationsverhältnis zwischen Rechtfertigungslehre und Kirchenbegriff statuierten, war diese Sichtweise den Zeitgenossen der CA noch fremd und deshalb für das 16. Jahrhundert in hohem Grad anachronistisch. Die konfessionelle

88 Klaus Unterburger, Das bayerische Konkordat von 1583. Die Neuordnung der päpstlichen Deutschlandpolitik nach dem Konzil von Trient und deren Bedeutung für das Verhältnis von weltlicher und geistlicher Gewalt, Stuttgart 2006 (Münchener Kirchenhistorische Studien 11), 110 -132; Hans-Jürgen Goertz, Pfaffenhaß und groß Geschrei. Die reformatorischen Bewegungen in Deutschland 1517-1529, München 1987; ders., Antiklerikalismus und Reformation. Ein sozialgeschichtliches Erklärungsmodell, in: ders., Antiklerikalismus und Reformation. Sozialgeschichtliche Untersuchungen, Göttingen 1995, 7-20. 
Aufladung der Fragen um Kirche und Amt, nach der es sich um heilsentscheidende Glaubensfragen handeln würde, ist späteren Datums, und damit, katholisch gesprochen, eher eine traditionsfremde, neuzeitliche Neologie oder, evangelisch gesprochen, eine unreformatorische Umdeutung des Anliegens der Reformatoren. ${ }^{89}$

Die Stellung der katholischen Theologie des 16. Jahrhunderts zur CA erweist sich so als wichtiger Indikator für das, was konfessionelle Abgrenzung damals hieß und was nicht. Die weitere Erforschung dieser Prozesse ist ein dringendes Postulat. Sie stellt zugleich Möglichkeiten für die Gegenwart bereit: Weder besteht das Wesen der Reformation darin, das allgemeine Priestertum der Gläubigen und die Freiheit und Mündigkeit der Laien gegenüber einer bevormundenden Sakralinstitution entdeckt $\mathrm{zu}$ haben, ${ }^{90}$ noch ist die Reformation ein Abfall von einer heilsentscheidenden, auf die bischöflichen Sukzession gegründeten, hierarchischen Heilsinstitution, deren Anerkennung über die Gültigkeit der Eucharistie und die Berechtigung des Begriffs „Kirche“ für eine Gemeinschaft erst entscheiden müsse. ${ }^{91}$ Zurückkehren zur CA und deren Anerkennung würde deshalb nicht nur die Realisierung von etwas bedeuten, was im 16. Jahrhundert nahelag, aber nie ganz realisiert wurde, sondern auch die produktive Aufnahme der Einsicht, dass die definitive Abgrenzung auf grundsätzlichem theologischen und ekklesiologischen Gebiet eben spät erfolgte und historisch kontingent ist und deshalb nicht zum Profil des Christlichen der eigenen Konfession gehören kann. Neben dieser Einsicht wäre zudem noch der Wille erforderlich, daraus dann Konsequenzen $\mathrm{zu}$ ziehen.

89 Klaus Unterburger, Trient im Fokus. Resonanzen der Gemeinsamen Erklärung in der Erforschung des Konzils von Trient, in: Bernd Oberdorfer/Thomas Söding (Hg.), Wachsende Zustimmung und offene Fragen. Die Gemeinsame Erklärung zur Rechtfertigungslehre im Licht ihrer Wirkung, Freiburg i.B./Basel/Wien 2019 (Quaestiones disputatae 302), 287-303.

90 Vgl. noch: Thomas Kaufmann, Erlöste und Verdammte. Eine Geschichte der Reformation, München 2016, 312-314.

91 Klaus Unterburger, Ecclesia de Eucharistia? Der Zusammenhang zwischen Eucharistiefeier und Kirchenstruktur in theologiegeschichtlicher Betrachtung, in: Stefan Kopp/Benedikt Kranemann (Hg.), Gottesdienst und Kirchenbilder. Theologische Neuakzentuierungen, Freiburg i.B. 2021 (Quaestiones disputatae), (im Druck). 